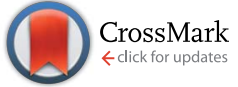

Cite this: RSC Adv., 2017, 7, 13886

Received 29th December 2016 Accepted 18th February 2017

DOI: 10.1039/c6ra28826j

rsc.li/rsc-advances

\section{Incorporating isosorbide as the chain extender improves mechanical properties of linear biodegradable polyurethanes as potential bone regeneration materials $\uparrow$}

\author{
Yufei Ma, ${ }^{\text {ab }}$ Juan Liu, ${ }^{\text {ab }}$ Min Luo, ${ }^{a}$ Juan Xing, ${ }^{a}$ Jinchuan Wu, ${ }^{a}$ Haobo Pan, ${ }^{b}$ \\ Changshun Ruan*b and Yanfeng Luo*a
}

\begin{abstract}
One key limitation to the application of linear biodegradable polyurethanes (LBPUs) in scaffold materials for bone regeneration is their insufficient mechanical properties. In this study, isosorbide (ISO), a rigid two-ring small diol is selected as a chain extender to produce a series of new poly (D,L-lactide)-based polyurethanes (ISO-PUs). It is confirmed that incorporating ISO as the chain extender can significantly reduce the crosslinking degree of ISO-PUs and thus increase their molecular weight and mechanical properties. ISO-PUs with $M_{n}$ values of $72.09 \mathrm{kDa}$ and $84.79 \mathrm{kDa}$ demonstrate higher tensile strength \& modulus (39.87 MPa \& 2.19 GPa and 42.68 MPa \& 2.57 GPa) than PDLLA with a $M_{n}$ of $100 \mathrm{kDa}(36.15 \mathrm{MPa} \& 2.07 \mathrm{GPa}$ ). All these results, together with the sound cytocompatibility of ISO-PUs with MC3T3-E1 cells based on the morphology observation and cell proliferation, suggest that ISO-PU should be a promising scaffold material for bone regeneration.
\end{abstract}

\section{Introduction}

Linear biodegradable polyurethanes (LBPUs) have been attracting lots of attention in biomedical applications due to their fascinating properties such as biocompatibility, ${ }^{1}$ biodegradability, ${ }^{2}$ mechanical properties ${ }^{3}$ and shape memory properties. $^{4,5}$ The most important one is that all performances of LBPUs can be tailored on the basis of the flexible molecular designability of LBPUs. Specifically, altering either the soft segment (polyester doils or polyether doils) or hard segment (diisocyanate, chain extender) in LBPUs will vary their performances to satisfy the versatile requirements in clinical applications. ${ }^{6-8}$ Despite these merits, the major clinical applications of existing LBPUs are focused on soft tissues such as the cardiovascular system and skin, among which the LBPUs are mainly based on polyether diol or polycaprolactone (PCL) diol. ${ }^{9}$ These LBPUs are highly elastic with low modulus of generally less than $60 \mathrm{MPa}$ even when the content of hard segments is up to $90 \mathrm{wt} \% .^{10,11}$ To extend the applications of LBPUs to bone

${ }^{a}$ Key Laboratory of Biorheological Science and Technology, Ministry of Education, Research Center of Bioinspired Materials Science and Engineering, College of Bioengineering, Chongqing University, Chongqing 400030, China. E-mail: yfluo@ cqu.edu.cn; Tel: +862365102509

${ }^{b}$ Center for Human Tissue and Organs Degeneration, Institute Biomedical and Biotechnology, Shenzhen Institutes of Advanced Technology, Chinese Academy of Sciences, Shenzhen 518055, China. E-mail: cs.ruan@siat.ac.cn; Tel: +86 755 86585250

$\dagger$ Electronic supplementary information (ESI) available. See DOI: $10.1039 / \mathrm{c} 6 \mathrm{ra} 28826 \mathrm{j}$ tissues, development of LBPUs with high modulus and strength is required.

Replacement of polyether diol or PCL diol by using more rigid poly(D,L-lactic acid) (PDLLA) diol can significantly enhance the tensile modulus of LBPUs from tens of megapascals to hundreds of megapascals, ${ }^{\mathbf{1 2 , 1 3}}$ which, however, cannot meet the requirement of bone scaffold materials either. According to the thorough review by Król, ${ }^{\mathbf{1 4}}$ employment of aromatic diisocyanate (toluene diisocyanate or methylenediphenyl diisocyanate) or introduction of urea groups into the backbone of LBPUs by using primary diamines instead of diols as the chain extender can improve the mechanical properties of LBPUs. This has been confirmed by our group and other groups. ${ }^{15}$ Unfortunately, the use of aromatic diisocyanate takes the stumble of getting toxic or potentially carcinogenic degradation products, ${ }^{\mathbf{1 6}}$ while primary diamines as chain extender brings the challenge of easily getting serious crosslinking during the synthesis of LBPUs because of the high reactivity of $-\mathrm{NH}_{2}$ with isocyanate. ${ }^{12}$ As a result, the mechanical properties of the obtained LBPUs are compromised and the degradation becomes difficult. ${ }^{13}$ To avoid the crosslinking, our previous studies employed piperazine (PPZ), a six-member ring secondary diamine to replace primary diamine such as 1,4-butanediamine (BDA) as the chain extender to synthesize PDLLA based LBPUs..$^{12,13,17}$ It was found that the crosslinking was significantly reduced and the tensile modulus and ultimate strength of the LBPUs were increased from $775 \mathrm{MPa}$ and 22.3 MPa for BDA to 1023 MPa and 34.7 MPa for PPZ, respectively, even when the content of hard segments was as low as only $12.12 \mathrm{wt} \%$. Nevertheless, there was still a little of 
crosslinking produced with the increase of the PPZ content. ${ }^{12}$ As a result, the molecular weight was decreased and the mechanical properties of the corresponding LBPUs were partially compromised. ${ }^{18,19}$

In this study, isosorbide (ISO), a small diol containing a rigid two-ring structure, was selected as the chain extender. Meanwhile, ISO was employed as a co-initiator of $\mathrm{D}, \mathrm{L}-$ lactide as well to prepare PDLLA diol (ISO-PDLLA). Consequently, new isosorbidebased polyurethanes (ISO-PUs) were prepared including soft segments (ISO-PDLLA) and hard segments (ISO and hexamethylene diisocyanate (HDI)) in the backbone of ISO-PUs. Based on this approach, twice introducing ISO into the polyurethane backbone is hypothesized to endow several potential advantages: (i) the special two-ring structure will be helpful to improve the rigidity of ISO-PUs backbones and further enhance their mechanical properties; (ii) employing diol instead of diamine to react with isocyanate should be beneficial to avoid crosslinking and elevate the molecular weight of ISO-PUs; (iii) ISO is also a natural small molecule drugs with good biocompatibility. ${ }^{20}$ It will not adversely affect the biocompatibility of ISO-PUs.

To verify the hypothesis, the chemical structure, crosslinking degree, molecular weight, glass transition temperature $\left(T_{\mathrm{g}}\right)$ and mechanical properties of ISO-PUs were well characterized through fourier transform infrared (FTIR), proton nuclear magnetic resonance $\left({ }^{1} \mathrm{H}\right.$ NMR) spectroscopy, gel permeation chromatography (GPC), differential scanning calorimetry (DSC) and tensile testing. Meanwhile, the morphology, proliferation and alkaline phosphatase (ALP) activity of MC3T3-E1 cell on the ISO-PUs films were performed to evaluate the potentiality of ISO-PUs for bone regeneration.

\section{Materials and methods}

\subsection{Materials}

D,L-Lactide, isosorbide, 1,6-hexamethylene diisocyanate (HDI), chloroform-d $\left(\mathrm{CDCl}_{3}\right)$, stannous octoate (Sn (Oct) $\left.)_{2}\right)$, dimethyl formamide, Triton X-100 and FITC-phalloidin (fluorescein isothiocyanate labeled phalloidin) were purchased from SigmaAldrich (California, USA). Cell counting kit-8 (CCK-8) and 4,6diamidino-2-phenylindole (DAPI) were purchased from Dojindo (Kumamoto, Japan). Alkaline phosphatase assay kit and BCIP/ NBT ALP color development kit were purchased from Beyotime (Shanghai, China). Live/dead viability/cytotoxicity kit was purchased from Thermo Fisher Scientific (California, USA). Ethanol, dichloromethane, chloroform, dimethylcarbinol and methylbenzene were purchased from Shanghai Lingfeng (Shanghai, China) and dehydrated with molecular sieve before used. PDLLA $\left(M_{\mathrm{n}}=100 \mathrm{kDa}\right)$, as the control in this study was purchased from Daigang (Jinan, China).

\subsection{Synthesis and characterization of ISO-PUs}

According to our previous reports, ${ }^{12,17}$ ISO-PUs were obtained by three steps as shown in Fig. 1. Firstly, PDLLA diols were prepared by ring-opening polymerization of D,L-lactide in the presence of $\mathrm{Sn}(\mathrm{Oct})_{2}$ as an initiator and ISO as a co-initiator at $140{ }^{\circ} \mathrm{C}$ for $24 \mathrm{~h}$ under vacuum (ISO/D,L-lactide $=1: 50 \mathrm{~mol} / \mathrm{mol}$,
$\operatorname{Sn}(\text { Oct })_{2} / \mathrm{D}, \mathrm{L}-$ lactide $\left.=1: 5000 \mathrm{~mol} / \mathrm{mol}\right)$. The obtained PDLLA diol (ISO-PDLLA) was purified thrice by co-precipitation of chloroform/absolute ethyl alcohol system at room temperature and dried under vacuum at room temperature to a constant weight. Subsequently, the purified ISO-PDLLA was terminated by HDI at $75{ }^{\circ} \mathrm{C}$ in a $250 \mathrm{~mL}$ three-necked flask equipped with a magnetic stirrer by using $\mathrm{Sn}(\mathrm{Oct})_{2}$ as the catalyst and anhydrous toluene as the solvent. Finally, ISO was added slowly for chain extension at $50{ }^{\circ} \mathrm{C}$ for $2 \mathrm{~h}$. The obtained mixture was purified by co-precipitation of toluene/ethanol system and dried under vacuum at room temperature to a constant weight, producing the desired ISO-PU polymers. In this process, the molecular weight of polyurethane was regulated by changing the ratio of ISO-PDLLA/HDI/ISO as listed in Table 1.

The ${ }^{1} \mathrm{H}$ NMR spectra were collected by using an AVANCE III 400 spectrometer (Bruker, Karlsruhe, Germany) with $\mathrm{CDCl}_{3}$ as the solvent. FTIR spectroscopy was performed on a VERTEX 70 (Bruker, Karlsruhe, Germany). The molecular weight and polydispersity index (PDI) of ISO-PUs were measured by GPC Viscotek 270 max (Malvern, Malvern, UK) with polystyrene as the standard. Three styrene-divinyl benzene (T-, D-, C-, HFIP-series) columns $(300 \times 8.0 \mathrm{~mm})$ were used in series with dimethyl formamide as the solvent at a flow rate of $1.0 \mathrm{~mL} \mathrm{~min}^{-1}$ at $25^{\circ} \mathrm{C}$. Thermal properties were measured from two cyclic heating and cooling scans using DSC Q20 (TA Instruments, New Castle, USA) under nitrogen atmosphere with a cooling rate of $10{ }^{\circ} \mathrm{C} \mathrm{min}^{-1}$ in the range -40 to $100^{\circ} \mathrm{C}$. The aluminum crucible was used to hold the sample and acted as blank control. The glass transition temperature $\left(T_{\mathrm{g}}\right)$ was determined from the second heating scan.

\subsection{Degree of crosslinking}

The crosslinking degrees $\left(G_{\mathrm{t}}\right)$ of ISO-PUs were investigated by using the method of solubility. Briefly, the ISO-PUs samples $\left(1 \mathrm{~g}\right.$, initial weight $\left.W_{0}\right)$ were immersed in $20 \mathrm{~mL}$ chloroform in a beaker with $300 \mathrm{rpm}$ stirring at $25^{\circ} \mathrm{C}$. After a predetermined time (i.e. 2, 4, 8, 16, and $24 \mathrm{~h}$ ), samples were removed from the solvent and dried to constant weight $\left(W_{t}\right)$. The degree of crosslinking $\left(G_{\mathrm{t}}\right)^{21,22}$ was calculated with eqn (1):

$$
G_{\mathrm{t}}(\%)=\left(\frac{W_{0}}{W_{t}}\right) \times 100
$$

\subsection{Mechanical properties}

According to the guidelines in ASTM D-638, ${ }^{23}$ ISO-PUs were injection-molded into dumbbell shape specimens at $150{ }^{\circ} \mathrm{C}$ (effective dimensions of $25 \mathrm{~mm} \times 4 \mathrm{~mm} \times 2 \mathrm{~mm}$ ), and the tensile stress-strain curves were collected by using an Instron 1121 instrument (Instron, Boston, USA) at $25^{\circ} \mathrm{C}, 50 \%$ humidity with the stretching strain rate of $5 \mathrm{~mm} \mathrm{~min}{ }^{-1}$. The tensile strength and modulus were calculated from the stress-strain curve and reported as the mean values $(n=3)$.

\subsection{Evaluation of cytocompatibility}

2.5.1. Cell culture. The cytocompatibility of ISO-PUs was evaluated by the co-culture assay of ISO-PUs films with murine calvarial preosteoblasts (MC3T3-E1, ATCC CRL-2594), while 


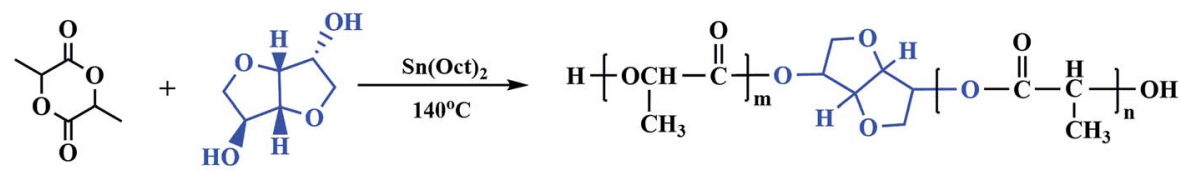

D,L-lactide Isosorbide (ISO)

PDLLA-Isosorbide-PDLLA Diol (ISO-PDLLA)

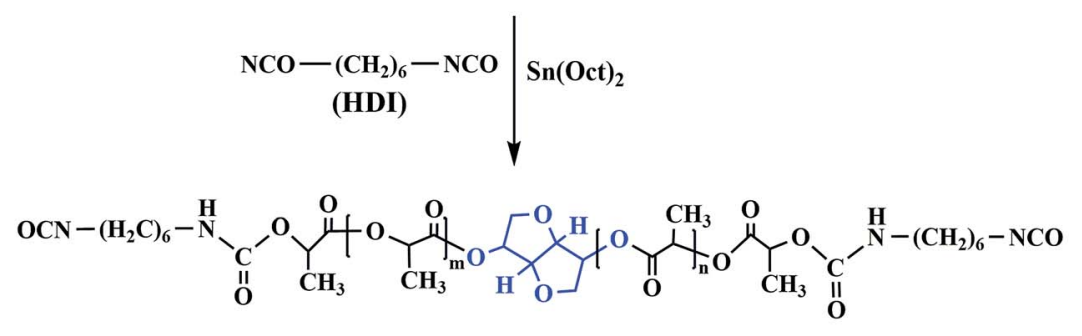

Isocyanate terminated prepolymer

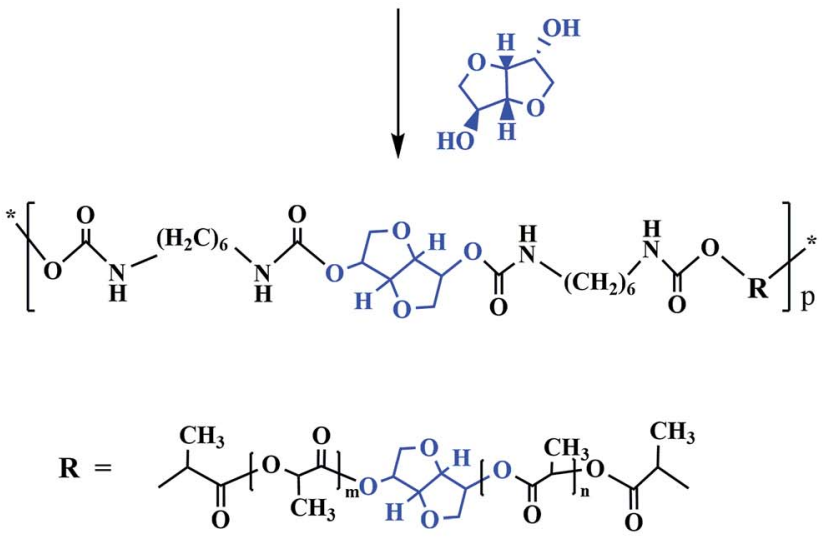

Isosorbide-based polyurethane (ISO-PUs)

Fig. 1 The schematic synthesis process of ISO-PUs.

Table 1 Molecular weights and $T_{\mathrm{g}}$ of various ISO-PUs and PDLLA

\begin{tabular}{|c|c|c|c|c|c|c|}
\hline Polymer $^{a}$ & $\begin{array}{l}\text { Molar ratio } \\
\text { ISO-PDLLA/HDI/ISO }\end{array}$ & $\begin{array}{l}\text { Segments contents }{ }^{b}(\mathrm{wt} \%) \\
\text { ISO-PDLLA/HDI/ISO }\end{array}$ & $M_{\mathrm{n}}(\mathrm{kDa})$ & $M_{\mathrm{w}}(\mathrm{kDa})$ & PDI & $T_{\mathrm{g}}\left({ }^{\circ} \mathrm{C}\right)$ \\
\hline LLA & - & - & 100.00 & 121.30 & 1.21 & - \\
\hline ISO-PU1.1 & $1.0 / 1.1 / 0.1$ & $97.31 / 2.49 / 0.20$ & 64.18 & 108.60 & 1.69 & $56.23 \pm 0.38$ \\
\hline ISO-PU1.3 & $1.0 / 1.3 / 0.3$ & $96.49 / 2.92 / 0.59$ & 72.09 & 128.36 & 1.78 & $58.42 \pm 0.51$ \\
\hline ISO-PU1.5 & $1.0 / 1.5 / 0.5$ & $95.69 / 3.34 / 0.97$ & 84.79 & 157.75 & 1.86 & $59.48 \pm 0.42$ \\
\hline
\end{tabular}

PDLLA was employed as the control polymer. The polymeric films were obtained by spin-coating (Laurell WS-400, California, USA) $50 \mu \mathrm{L}$ of $4 \mathrm{wt} \%$ polymer solution in chloroform on clean glass slides (diameter $=14 \mathrm{~mm}$ ) and then allowing for slow evaporation at $25{ }^{\circ} \mathrm{C}$ for $96 \mathrm{~h}$. Before cell culture, the films were sterilized by ultraviolet irradiation for $30 \mathrm{~min}$. All samples were placed in 24-well cell culture plates for cell seeding and culturing. Cells were seeded onto polymer substrates at a density of $1 \times 10^{4}$ cells per well for all experiments, except for specified descriptions.

2.5.2. Live/dead staining. The Live/dead staining was utilized to perform the primary cytotoxicity of ISO-PUs. After seeding MC3T3-E1 cells on polymeric films for $24 \mathrm{~h}$ of culture, the samples was stained with Live/dead viability/cytotoxicity kit for $30 \mathrm{~min}$ at $25{ }^{\circ} \mathrm{C}$ and the fluorescent images were observed with a fluorescence microscopy (BX53, Olympus, Tokyo, Japan). 
2.5.3. Cell morphology. The cell morphology on films was observed by means of fluorescent staining as established previously. For staining, briefly, MC3T3-E1 cells were seeded on films $(n=3)$ for $24 \mathrm{~h}$ of culture, and then fixed by $4 \%$ (wt/v) paraformaldehyde at $4{ }^{\circ} \mathrm{C}$ for $30 \mathrm{~min}$ and permeabilized with $0.1 \%$ Triton X-100 in PBS solution for $5 \mathrm{~min}$. The cytoskeleton and nuclei were stained with FITC-phalloidin and DAPI, respectively. The cytoskeleton and cell nuclei were detected with a confocal laser scanning microscope (Leica TCS SP8, Wetzlar, Germany). Cell spreading area was measured by Image (NIH) using the active contours algorithm. ${ }^{24}$

2.5.4. Cell proliferation. The relative cell viability and proliferation was determined using a cell counting assay. ${ }^{25}$ The MC3T3-E1 cells were seeded onto ISO-PUs and PDLLA films in the modified Eagle's medium alpha ( $\alpha$-MEM, GE Hyclone, Logan, USA) supplemented with $10 \%$ fetal calf serum and $1 \%$ penicillin-streptomycin. They were incubated under a $\mathrm{CO}_{2}(5 \%)$ atmosphere at $37^{\circ} \mathrm{C}$ and the culture medium was changed every 2 days. After 1, 3, 5 and 7 days of culture on the films $(n=6)$, CCK-8 solutions were added to each well and incubated for $2 \mathrm{~h}$, and the optical density (OD) was measured by a microplate reader (Bio-Rad 550, California, USA) at $450 \mathrm{~nm}$ to determine the viability of MC3T3-E1 cells in comparison with the control.

2.5.5. Alkaline phosphatase assay (ALP assay). MC3T3-E1 cells were seeded on polymeric films at a density of $1 \times 10^{5}$ cells per well to determine the levels of ALP activity. After 4, 7 and 14 days of co-culture, the cells were washed thrice with PBS and lysed in 0.2 vol\% Triton X-100. The ALP activity was determined using a colorimetric assay with an ALP reagent containing $p$-nitrophenyl phosphate ( $p$-NPP) as the substrate (Beyotime, Shanghai, China). The absorbance of $p$-nitrophenol was monitored at $405 \mathrm{~nm}$. The intracellular total protein content was determined using the MicroBCA protein assay kit (Thermo Pierce, California, USA), and the ALP activity was normalized to the total protein content. For ALP staining, the samples cultured for 14 days were rinsed twice with PBS, fixed by $4 \%(\mathrm{wt} / \mathrm{v})$ paraformaldehyde at $4{ }^{\circ} \mathrm{C}$ for $30 \mathrm{~min}$, and rinsed with ultra-pure water for $45 \mathrm{~s}$. Then the samples were stained with BCIP/NBT alkaline phosphatase color development kit (Beyotime, Shanghai, China) followed the manufacturer's protocol and pictures were taken by microscopy (BX 53, Olympus, Tokyo, Japan).

\subsection{Statistical analysis}

One-way single factor analysis of variance (ANOVA) was used to analyze the statistical significance of experimental results. Experimental data were expressed as means $\pm \operatorname{SD}(n \geq 3)$, and a paired $t$-test (Student's $t$-test) was performed with $p<0.05$ considered to be statistically significant.

\section{Results and discussion}

\subsection{Synthesis and characterization of ISO-PDLLA diol and} ISO-PUs

As shown in Fig. 1, ISO as the co-initiator was first employed to synthesize PDLLA diol (ISO-PDLLA) via ring-opening polymerization of $\mathrm{D}, \mathrm{L}-\mathrm{lactide}$. ISO-PDLLA was then employed to synthesize a series of ISO-PUs by regulating the molar ratio of ISO-PDLLA/HDI/ISO, i.e. 1.0/1.1/0.1, 1.0/1.3/0.3 and 1.0/1.5/0.5 (Table 1). The obtained ISO-PUs were coded as ISO-PU1.1, ISO-PU1.3, ISO-PU1.5, respectively.

To verify the successful synthesis of ISO-PDLLA and ISO-PUs, FTIR was first employed to characterize the polymers. The typical FTIR spectra of ISO-PDLLA and ISO-PU1.3 were illustrated in Fig. 2. The spectrum of ISO-PDLLA exhibited the characteristic peaks of $\mathrm{OH}$ stretching vibration $\left(3506 \mathrm{~cm}^{-1}\right)$ and ester $\mathrm{C}=\mathrm{O}$ stretching vibration $\left(1753 \mathrm{~cm}^{-1}\right)$. Compared with that of ISO-PDLLA, the spectrum of ISO-PU1.3 demonstrated three new absorption peaks at $3403 \mathrm{~cm}^{-1}, 1657 \mathrm{~cm}^{-1}$ and 1524 $\mathrm{cm}^{-1}$, which should be attributed to NH stretching vibration, and amide I and amide II bands in urethane amide, respectively. ${ }^{12}$ The characteristic peaks of ISO were not observed possibly because of the low contents of ISO in both ISO-PDLLA and ISO-PU1.3.

To further verify the existence of ISO in both ISO-PDLLA and ISO-PUs, the ${ }^{1} \mathrm{H}$ NMR spectra of ISO-PDLLA and ISO-PU1.3 were collected and shown in Fig. 3a and b, respectively. In Fig. 3a, peak a belonged to the chemical shifts of $-\mathrm{CH}$ protons within the PDLLA segments, while peaks $\mathrm{a}^{\prime}$ and $\mathrm{a}^{\prime \prime}$ corresponded to the terminal $\mathrm{CH}$ protons of lactyl units connecting with $-\mathrm{OH}$ and the $\mathrm{CH}$ protons of lactyl units connecting with ISO. Those $\mathrm{CH}_{3}$ protons from lactyl units were collectively observed by peaks $b$, $b^{\prime}$ and $b^{\prime \prime}$. These peaks were observed as well by the previously published results. ${ }^{\mathbf{1 2 , 1 3}}$ In addition to the peaks from PDLLA segments, other peaks from ISO were observed. Specifically, peak c was assigned to the $\mathrm{CH}$ protons of ISO connecting with the ester groups, while $\mathrm{d}$ and e were assigned to the $\mathrm{CH}_{2}$ and $\mathrm{CH}$ groups connecting with the ether groups in ISO. Similar peaks from ISO were partially observed by van Velthoven et al. as well. ${ }^{26}$ Peak c was noticed to overlap with peak $\mathrm{a}^{\prime}$. To confirm these assignments, the integrals of $\mathrm{a}^{\prime \prime}\left(I_{\mathrm{a}^{\prime \prime}}\right), \mathrm{c} \& \mathrm{a}^{\prime}\left(I_{\mathrm{c}+\mathrm{a}^{\prime}}\right)$ and $\mathrm{d} \& \mathrm{e}$ $\left(I_{\mathrm{d}+\mathrm{e}}\right)$ were calculated. Since the integral of $\mathrm{a}^{\prime}\left(I_{\mathrm{a}^{\prime}}\right)$ should be theoretically identical to that of $\mathrm{a}^{\prime \prime}\left(I_{\mathrm{a}^{\prime \prime}}\right)$, the integral of $\mathrm{c}\left(I_{\mathrm{c}}\right)$

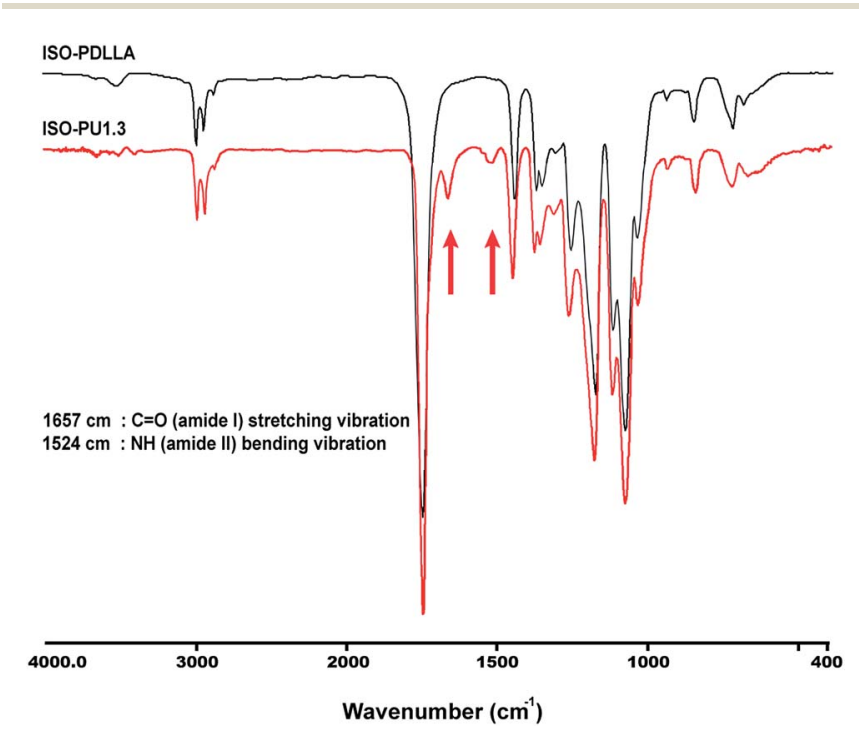

Fig. 2 Typical FTIR spectra of ISO-PDLLA and ISO-PU1.3. 
(a)

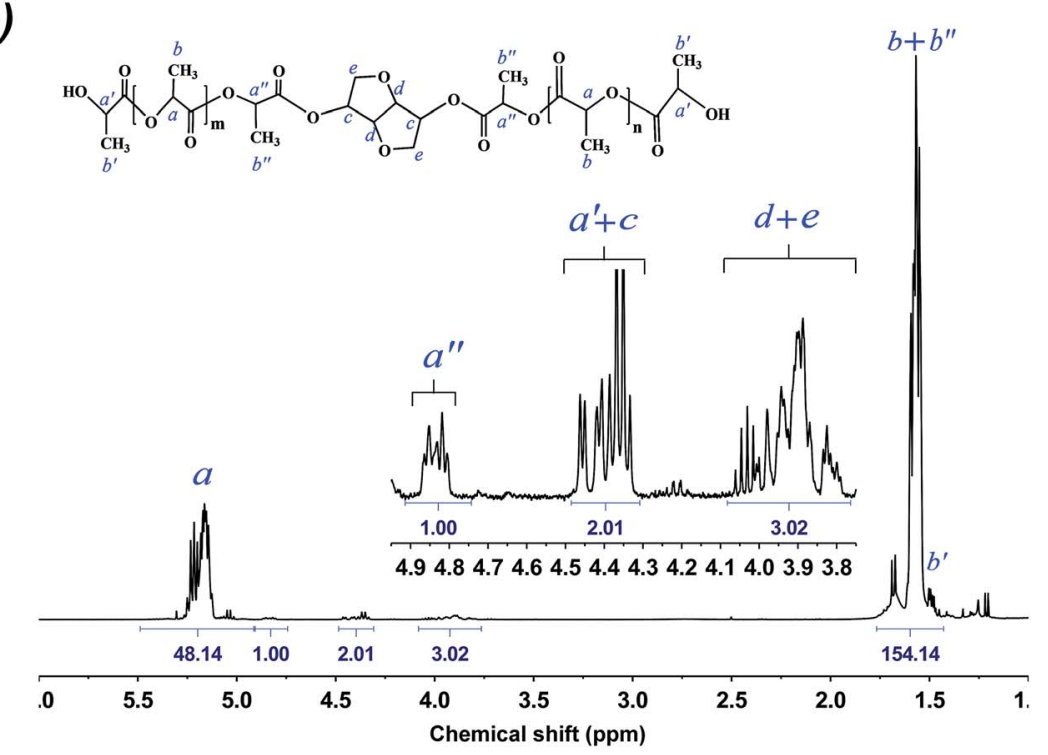

(b)

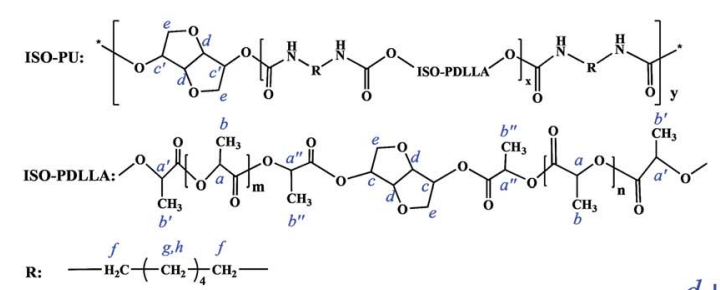
$I_{\mathrm{c}}=I_{\mathrm{c}+\mathrm{a}^{\prime}}-I_{\mathrm{a}^{\prime \prime}}=2.01-1.00=1.01$. Obviously, $I_{\mathrm{d}+\mathrm{e}}$ was almost thrice of $I_{\mathrm{c}}\left(I_{\mathrm{d}+\mathrm{e}} / I_{\mathrm{c}}=3.02 / 1.01 \approx 3 / 1\right)$, conforming to the stoichiometric ratio in ISO molecules. These results revealed that ISO has been successfully inserted into the backbone of PDLLA diol. Moreover, based on $I_{\mathrm{a}}(=48.14)$ and $I_{\mathrm{a}^{\prime \prime}}(=1.00)$ (or $I_{\mathrm{c}}$ (=1.01)), the $M_{\mathrm{n}}$ of ISO-PDLLA could be calculated by using eqn (2):

$$
M_{\mathrm{n}}=\left[\frac{I_{\mathrm{a}}}{I_{\mathrm{a}^{\prime \prime}} \times \frac{1}{2}}+2\right] \times 72+146=\left[\frac{I_{\mathrm{a}}}{I_{\mathrm{c}} \times \frac{1}{2}}+2\right] \times 72+146
$$

where, 72 is the molecular weight of lactyl repeating units within PDLLA blocks and 146 is the molecular weight of ISO. The calculated $M_{\mathrm{n}}$ was about $7222 \mathrm{~g} \mathrm{~mol}^{-1}$, which is consistent with the reported result that, when the molar ratio of co-

(a)

Crosslinking degree of various ISO-PUs

\begin{tabular}{cccccc}
\hline \multirow{2}{*}{ Sample } & \multicolumn{5}{c}{ Crosslinking degree (\%) } \\
\cline { 2 - 6 } & $2 \mathrm{~h}$ & $4 \mathrm{~h}$ & $8 \mathrm{~h}$ & $16 \mathrm{~h}$ & 24 \\
\hline ISO-PU1.1 & 0 & 0 & 0 & 0 & 0 \\
ISO-PU1.3 & 3.15 & 0 & 0 & 0 & 0 \\
ISO-PU1.5 & 12.26 & 4.58 & 2.67 & 0 & 0 \\
\hline
\end{tabular}

(b)

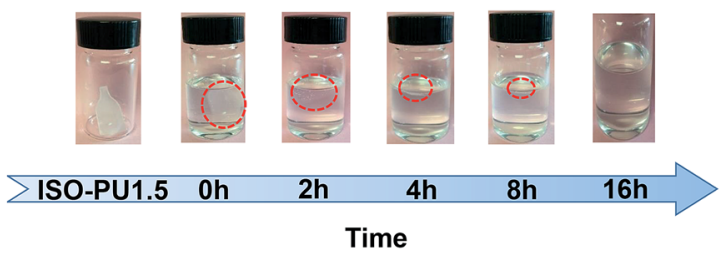

Fig. 4 (a) The crosslinking degree of ISO-PUs; (b) dissolution of ISOPU1.5 in chloroform. 
initiator to D,L-lactide was $1 / 50$, the obtained PDLLA diol could get a $M_{\mathrm{n}}$ value of about $7.0 \mathrm{kDa} \cdot{ }^{13}$

After ISO-PDLLA was reacted with HDI and ISO (chain extender), the peaks from the terminal $\mathrm{CH}_{3}$ (peak b') and $\mathrm{CH}$ (peak $\mathrm{a}^{\prime}$ ) in ISO-PDLLA almost disappeared in ISO-PUs, and new peaks $\mathrm{f}, \mathrm{g}$ and $\mathrm{h}$ were observed (Fig. $3 \mathrm{~b}$ ). Peak $\mathrm{f}$ was assigned to the terminal $\mathrm{CH}_{2}$ protons of HDI connecting with the urethane groups, whereas peak $\mathrm{g}$ and $\mathrm{h}$ were attributed to the inner four
$\mathrm{CH}_{2}$ of HDI. These assignments could be further verified by the stoichiometric ratio of $I_{\mathrm{g}+\mathrm{h}}$ to $I_{\mathrm{f}}$, i.e. $I_{\mathrm{g}+\mathrm{h}} / I_{\mathrm{f}}=6.36 / 3.14 \approx 2 / 1$. It was worthy of noting that the chain extension of ISO-PDLLA by ISO resulted in higher ISO content in ISO-PU polymer than in ISO-PDLLA. Specifically, the molar ratio of ISO (including both the co-initiator ISO and the chain extender ISO) to ISO-PDLLA could be calculated based on the ratio of $\left(I_{\mathrm{c}+\mathrm{d}+\mathrm{e}} / 4\right) / I_{\mathrm{a}^{\prime \prime}}$. In ISOPU1.3, the ratio was close to $1.32 / 1\left(I_{\mathrm{c}+\mathrm{d}+\mathrm{e}} / 4=(1.58+3.72) / 4\right.$
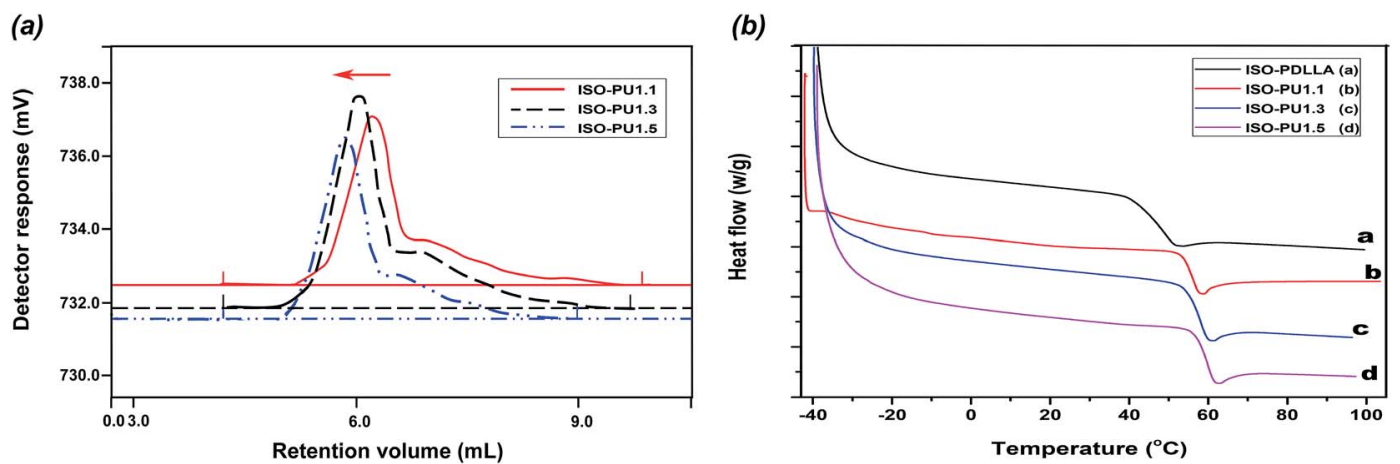

Fig. 5 (a) The typical GPC curves of ISO-PUs; (b) the typical DSC curves of ISO-PDLLA and ISO-PUs.

(a)

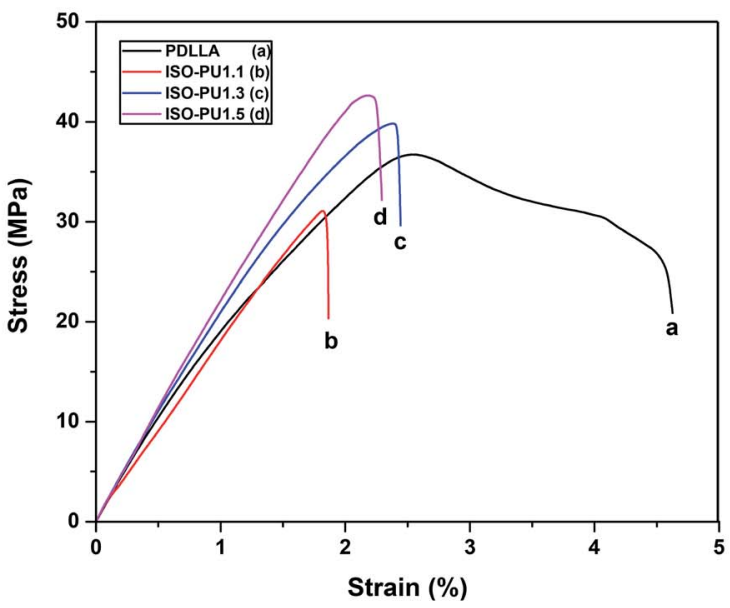

(c)

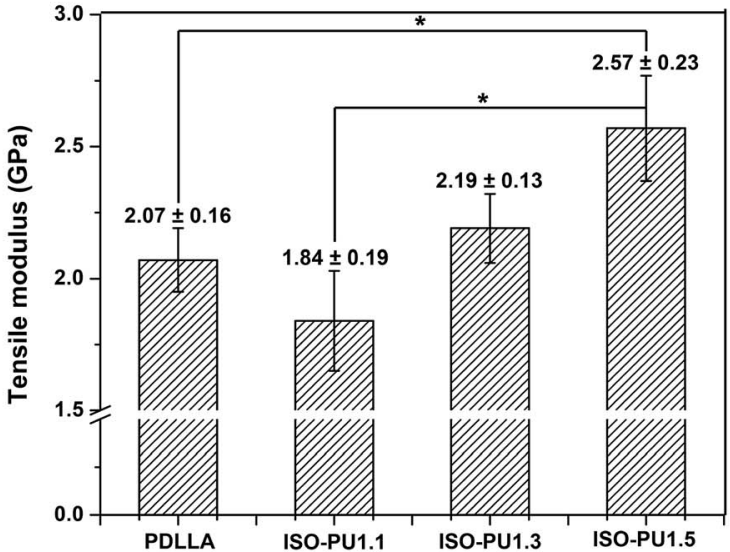

(b)

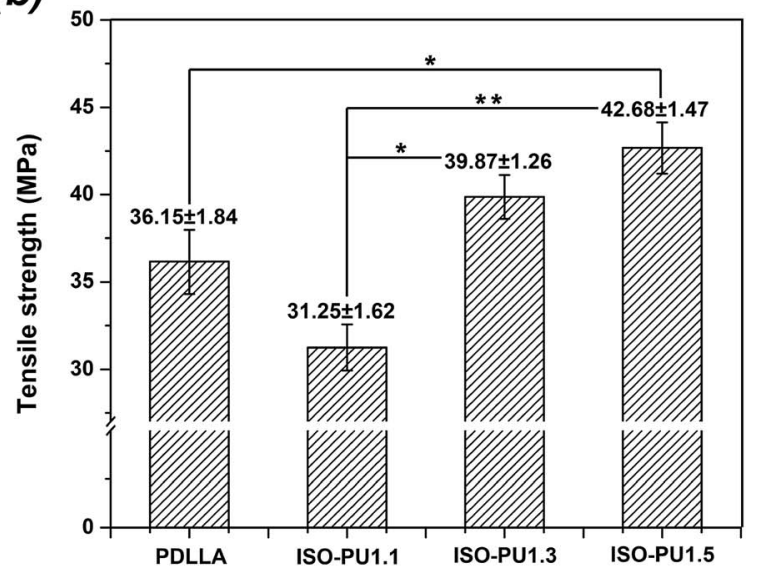

(d)

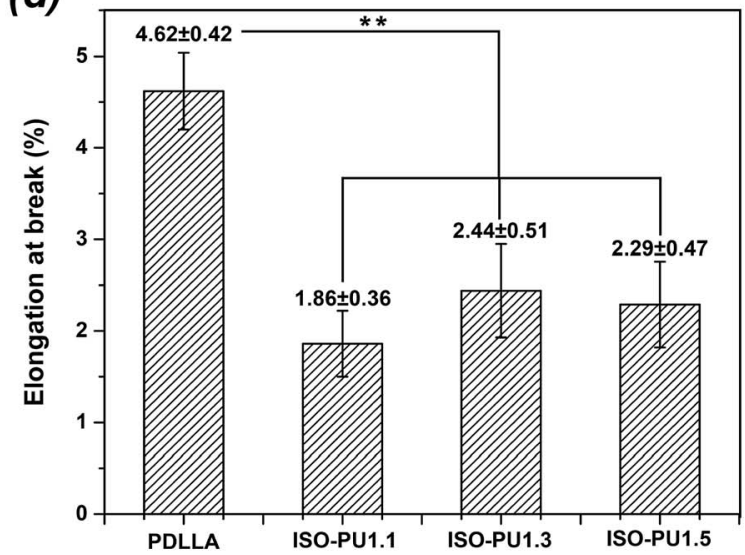

Fig. 6 The mechanical properties of PDLLA and ISO-PUs: (a) the typical stress-strain curves; (b) tensile strength; (c) tensile modulus; (d) elongation at break (*means $P<0.05, * *$ means $P<0.01$ ). 
$\left.\approx 1.32 ; I_{\mathrm{a}^{\prime \prime}}=1.00\right)$ whereas in ISO-PDLLA, the ratio was close to $1 / 1\left(I_{\mathrm{c}+\mathrm{d}+\mathrm{e}} / 4=(2.01+3.02-1.00) / 4 \approx 1 ; I_{\mathrm{a}^{\prime \prime}}=1.00\right)$, indicating higher ISO content in ISO-PU1.3. All above results confirm that ISO as the chain extender has been successfully inserted to get the desired ISO-PUs.

\subsection{Degree of crosslinking}

It is known that one challenge for preparing LBPUs as an implanted material is to avoid crosslinking during the synthesis process. To confirm our hypothesis that introducing ISO as the chain extender may reduce crosslinking, the crosslinking degrees of ISO-PUs were investigated by using the method of solubility. The polymer with lower crosslinking degree will dissolve more rapidly in a suitable solvent. As shown in Fig. 4a, ISO-PU1.1, ISO-PU1.3 and ISO-PU1.5 completely dissolved in chloroform after immersion of $2 \mathrm{~h}, 4 \mathrm{~h}$ and $16 \mathrm{~h}$, respectively, demonstrating negligible crosslinking. As reported previously, piperazine (PPZ) as the chain extender could reduce the crosslinking of LBPUs when compared with BDA; PPZ extended PU (PPZ-PUs) could completely dissolve in chloroform after immersion of $24 \mathrm{~h}$ when the molar ratio of PDLLA diol/HDI/PPZ was up to $1.0: 1.4: 0.4$, beyond of which, however, the crosslinking degree sharply increased so that the obtained PPZ-PUs became insoluble in chloroform. ${ }^{17}$ On the contrary, in this present study, even when the molar ratio of ISO-PDLLA/HDI/ISO was as high as $1.0: 1.5: 0.5$, most of the ISO-PU1.5 could dissolve in chloroform after immersion of $8 \mathrm{~h}$ though a complete dissolution took place after $16 \mathrm{~h}$ (Fig. $4 \mathrm{~b}$ and $\mathrm{S} 1 \dagger$ ). Therefore, it may be concluded that the selection of ISO as the chain extender could indeed deal with the problem of crosslinking during the synthesis of LBPUs.

\subsection{The molecular weight and polydispersity index (PDI)}

The typical GPC curves of various ISO-PUs were shown in Fig. 5a (Fig. $\mathrm{S} 2 \dagger$ ) and the detailed molecular weight and PDI values were summarized in Table 1 . It was seen that, with the increase of ISO-PDLLA/HDI/ISO from $1.0: 1.1: 0.1$ to $1.0: 1.5: 0.5$, the $M_{\mathrm{n}}$ values of ISO-PUs increased from $64.18 \mathrm{kDa}$ to $84.79 \mathrm{kDa}$ and the PDI values increased from 1.69 to 1.86 . This continuous increase of molecular weight should be contributed by the negligible crosslinking during chain extension by ISO (Table 1 , Fig. 4b). Conversely, the highest $M_{\mathrm{n}}$ of PPZ-PUs was seen as only

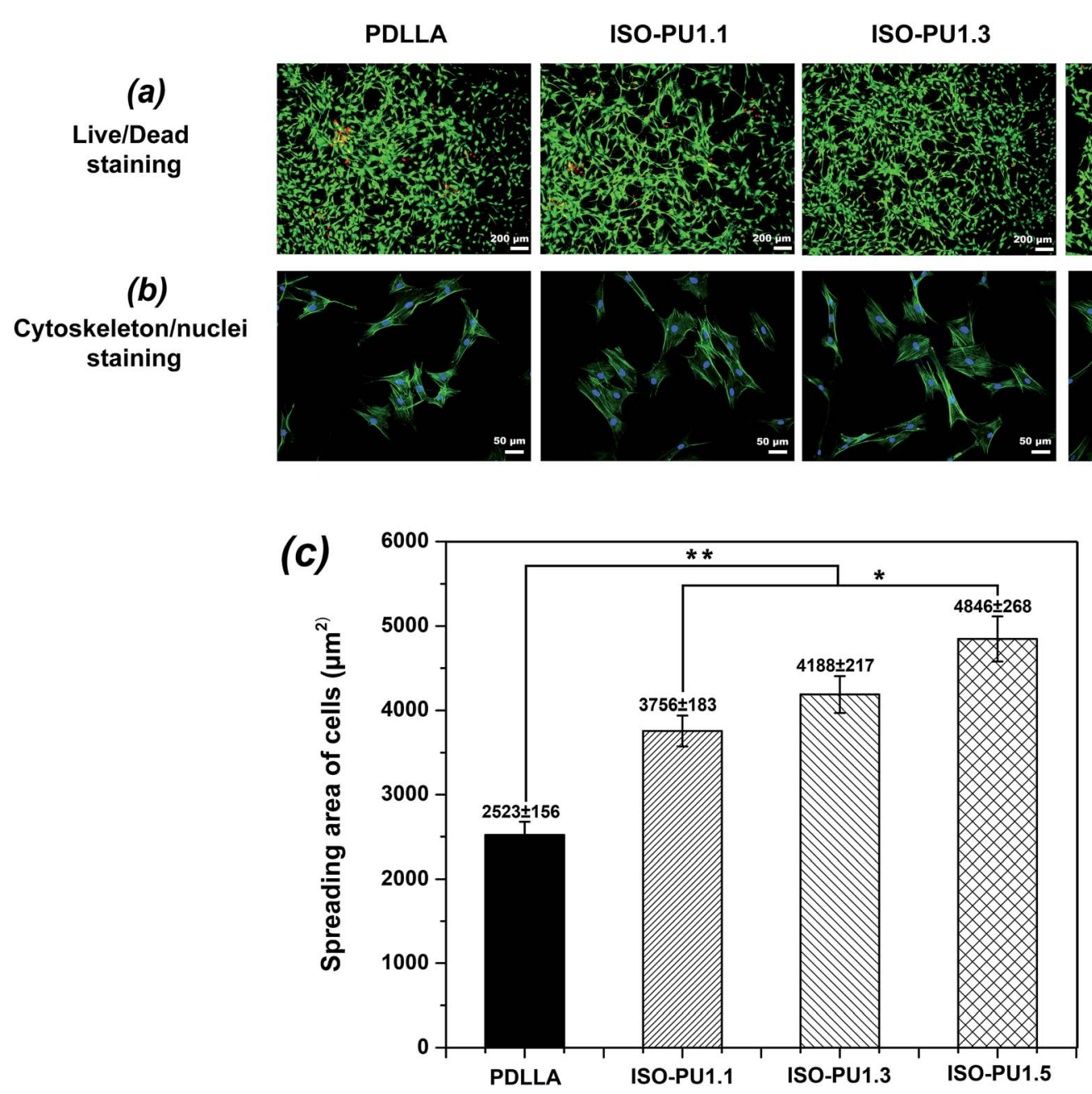

Fig. 7 (a) Live/dead staining for cell after seeding of $24 \mathrm{~h}$, green fluorescence indicating cells alive while the red visualizing dead cells; (b) cell morphology after seeding of $24 \mathrm{~h}$; (c) cell spreading areas $(n=200)$ after seeding of $24 \mathrm{~h}$ (*means $P<0.05, * *$ means $P<0.01$ ). 
$50.81 \mathrm{kDa}$ due to the existence of crosslinking. ${ }^{17-19}$ It should be especially noted that ISO-PU1.5 had the highest PDI and $M_{\mathrm{w}}$ values of 1.86 and $157.75 \mathrm{kDa}$, respectively, which should be responsible for its slow dissolution in chloroform (Fig. 4b). Again, we could conclude that ISO as chain extender can improve the molecular weight of ISO-PUs by reducing the degree of crosslinking.

\subsection{Thermal properties}

The DSC analysis was executed to characterize the thermal behaviors of ISO-PDLLA and corresponding ISO-PUs, and the results were summarized in Fig. $5 \mathrm{~b}$ and Table 1 . Compared to the $T_{\mathrm{g}}$ of ISO-PDLLA $\left(48.37^{\circ} \mathrm{C}\right)$, the $T_{\mathrm{g}}$ of all ISO-PUs increased significantly, suggesting that the more ISO-PDLLA segments were embedded in the backbone of the ISO-PUs to confirm the successful synthesis of ISO-PUs again. Moreover, with the increase of ISO-PDLLA/HDI/ISO from $1.0: 1.1: 0.1$ to $1.0: 1.5: 0.5$, the $T_{\mathrm{g}}$ of ISO-PUs increased from $56.23{ }^{\circ} \mathrm{C}$ to $59.48{ }^{\circ} \mathrm{C}$. According to Gnanarajan's and Liu's works, ${ }^{27,28}$ the chemical structure of polyurethane played a key role in influence of its $T_{\mathrm{g}}$, especially its hard-soft phase separate in polyurethane. Therefore, the increase of $T_{\mathrm{g}}$ might be attributed to the multi-segmental structure of ISO-PUs, in which, HDI/ISO as the hard segments provided an abundance of rigid ISO rings and rigid urethane groups for the ISO-PUs, which restrict the mobility of soft segments. Also, with the increase of hard segments contents, the more hydrogen bonds might formed by the carbonyl groups in ISO-PDLLA diol and the -NH- in urethane groups, further limited the rotation of soft segments and increased $T_{\mathrm{g}}{ }^{29,30}$

\subsection{Mechanical properties}

Sufficient mechanical properties are essential for a scaffold material to support the repair process of the defect bone tissue. ${ }^{31}$ Compared with other traditional biodegradable materials such as biodegradable metals and ceramics, biodegradable polymers are relatively weak in mechanical properties. Among various biodegradable polymers, PDLLA is one of the most popular biodegradable polymers in orthopedic devices, whose elastic modulus was reported to be around $2 \mathrm{GPa}^{32,33}$ In this study, PDLLA with a $M_{\mathrm{n}}$ value of $100 \mathrm{kDa}$ was chosen as a reference for evaluating the mechanical properties of ISO-PUs.

Fig. 6a illustrated the tensile stress-strain curves of PDLLA and ISO-PUs. The corresponding ultimate tensile strength $(\sigma)$, tensile modulus $\left(E_{\mathrm{t}}\right)$, and elongation at break $\left(\varepsilon_{\mathrm{b}}\right)$ were presented in Fig. 6b-d, respectively. It was observed that the tensile strength and modulus of all ISO-PUs were more than $30 \mathrm{MPa}$ and $1.8 \mathrm{GPa}$, respectively, which were obviously higher than those of previously reported LBPUs including PPZ-PUs. ${ }^{12}$ In particular, the tensile strength of ISO-PUs significantly increased from $31.25 \pm 1.62 \mathrm{MPa}$ for ISO-PU1.1 to $42.68 \pm$ 1.47 MPa for ISO-PU1.5. On the other hand, the tensile modulus of ISO-PUs kept increasing from $1.84 \pm 0.19 \mathrm{GPa}$ for ISO-PU1.1 to $2.57 \pm 0.23 \mathrm{GPa}$ for ISO-PU1.5. Compared with PDLLA, both ISO-PU1.3 and ISO-PU1.5 demonstrated higher tensile strength and modulus though their elongation at break was slightly smaller than that of PDLLA. All these results are well consistent (a)

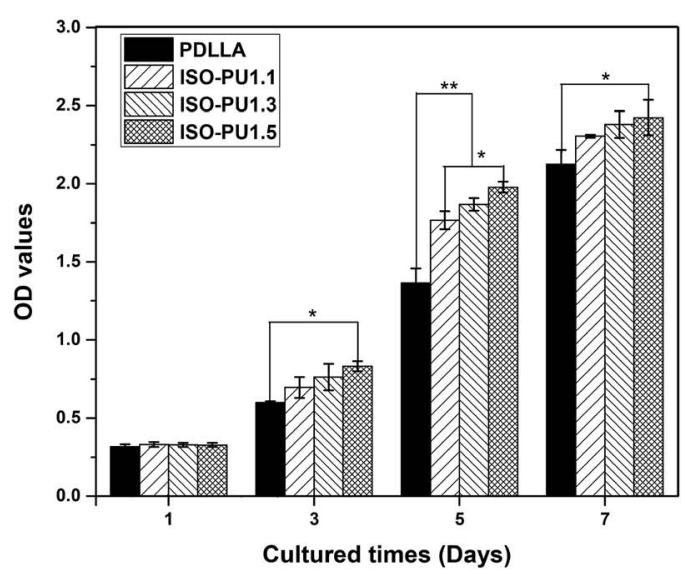

(c)

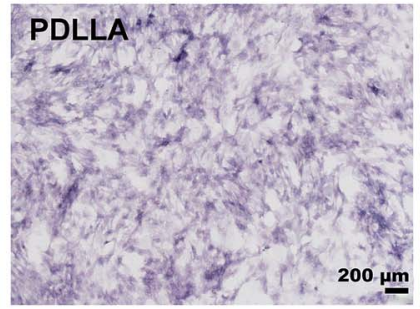

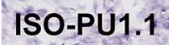

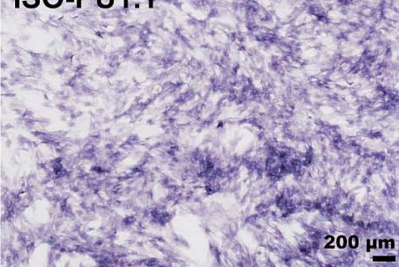

(b)
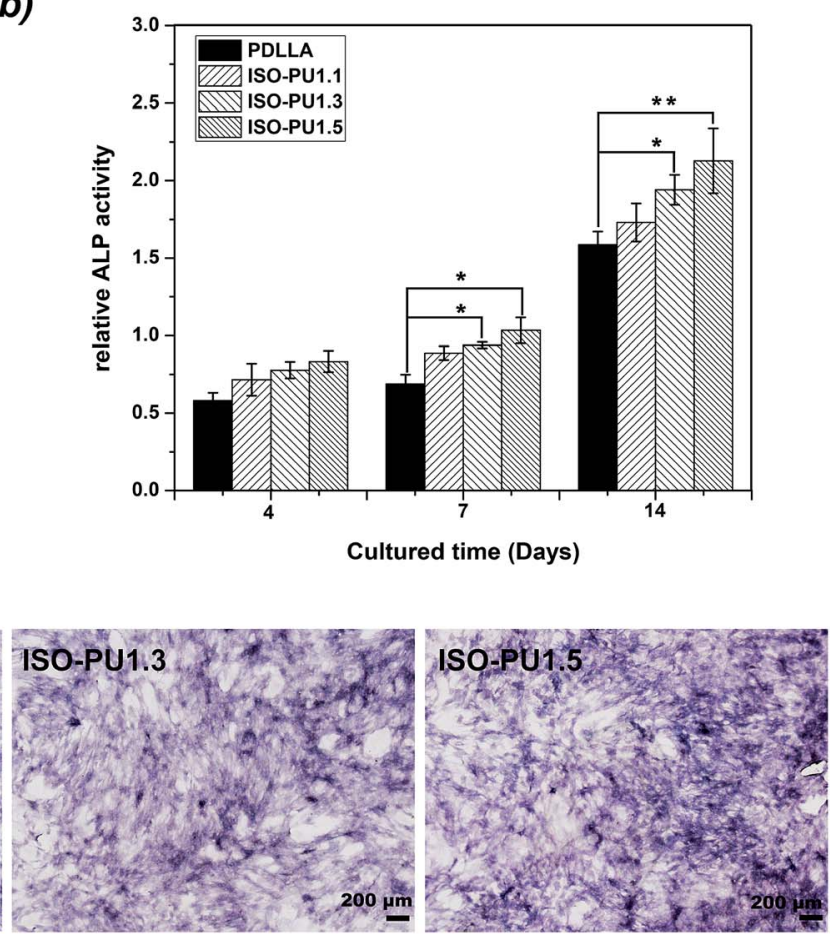

Fig. 8 (a) Cell proliferation by CCK-8 after seeding of 1, 3, 5, 7 days; (b) the relative ALP activity of cell on PDLLA and ISO-PUs films; (c) the coloration of ALP activity (deep blue) on PDLLA and ISO-PUs films at $14 \mathrm{~d}$ (*means $P<0.05$, **means $P<0.01$ ). 
with our hypothesis that the rigid double-ring structure of ISO and the ease control of crosslinking by using ISO can enhance the mechanical properties of ISO-PUs.

\subsection{Evaluation of cellular compatibility}

In addition to sufficient mechanical properties, sound cytocompatibility is indispensable as well. Firstly, the viability and morphology of MC3T3-E1 cells on various polymeric films for $24 \mathrm{~h}$ of culture were detected as shown in Fig. 7. Live/dead staining (Fig. 7a) revealed that there were fewer red dead cells on ISO-PUs than those on PDLLA, suggesting the better primary compatibility of ISO-PUs than PDLLA. This was further confirmed by the results of morphology of MC3T3-E1 cells (Fig. 7b and c). The cells on ISO-PUs films spread better than those on PDLLA films $\left(2523 \pm 156 \mu \mathrm{m}^{2}\right)$. Moreover, the cell areas increased from $3756 \pm 183 \mu \mathrm{m}^{2}$ to $4846 \pm 268 \mu \mathrm{m}^{2}$ with the increase of hard segments content from $1.0: 1.1: 0.1$ to $1.0: 1.5: 0.5$. This should be due to the abundant urethane/ urea groups in ISO-PUs, which are hydrophilic and proved to promote cell adhesion and spread. ${ }^{34}$

Furthermore, the better primary compatibility and spreading on ISO-PUs promoted better cell proliferation than that on PDLLA films, especially after cell seeding of 3 days (Fig. 8a). The similarly low cell proliferation between PDLLA and ISO-PUs on day 1 should be attributed to the fact that the cells at this stage were primarily expressing proteins to adapt cell adhesion, spread and subsequent cell proliferation. As a result, the cell proliferation on ISO-PUs was promoted with the increase of hard segments as well. Based on the cell morphology and cell proliferation, ISO-PUs demonstrated better cytocompatibility with MC3T3-E1 cells than PDLLA.

In addition, the initial osteo-differentiation of MC3T3-E1 cells on PDLLA and ISO-PUs films was detected with ALP activity at 4, 7 and 14 days of culture. As illustrated in Fig. 8b, the relative ALP activity for all groups increased during the testing cycle. Meanwhile, the ALP activity of MC3T3-E1 cells on ISO-PUs improved significantly compared to PDLLA at 14 days, except for ISO-PU1.1. These results were further confirmed by the stained images of ALP-positive areas of MC3T3-E1 cells on polymeric films for 14 days as shown in Fig. 8c. It noted that the ALP-positive areas of PDLLA were the least and the ALP-positive areas of ISO-PUs improved with the increase of hard segments content. Therefore, it could be concluded that ISO-PUs had certain osteogenic capability, which would be beneficial for bone regeneration.

\section{Conclusions}

A series of novel biodegradable ISO-PUs with ISO as the chain extender were synthesized successfully. The results revealed that incorporating ISO as chain extender could significantly reduce the degree of crosslinking and increase the molecular weight of ISO-PUs. Moreover, the obtained ISO-PUs presented excellent tensile strength and modulus and their mechanical properties could further improve with increase of hard segments contents. In addition, the ISO-PUs also demonstrated good cytocompatibility and osteo-differentiation with osteoblasts, suggesting a potentially ideal biomaterial for bone regeneration.

\section{Acknowledgements}

This work was supported by grants from National High-Tech Research and development program (No. 2015AA020316), the National Natural Science Foundation of China (No. 31370946 and No. 11532004), the Development of Strategic Emerging Industries of Shenzhen Project (JCYJ20140417113430596 and CXZZ20140417113430716) and the Shenzhen Peacock Program (110811003586331).

\section{References}

1 J. Zhang, T. Woodruff, R. Clark, D. Martin and R. Minchin, Acta Biomater., 2016, 41, 264-272.

2 L. Tan, Q. Su, S. Zhang and H. Huang, RSC Adv., 2015, 5, 80884-80892.

3 L. Huang, N. Yi, Y. Wu, Y. Zhang, Q. Zhang, Y. Huang, Y. Ma and Y. Chen, Adv. Mater., 2013, 25, 2224-2228.

4 Y. Heo and H. Sodano, Adv. Funct. Mater., 2014, 24, 52615268.

5 J. Xing, Y. Ma, M. Lin, Y. Wang, H. Pan, C. Ruan and Y. Luo, Colloids Surf., B, 2016, 146, 431-441.

6 P. Chen, H. Liao, S. Hsu, R. Chen, M. Wu, Y. Yang, C. Wu, M. Chen and W. Su, RSC Adv., 2015, 5, 6932-6939.

7 Y. Wang, F. Fang, Y. Wu, X. Ai, T. Lan, R. Liang, Y. Zhang, N. Trishul, M. He, C. You, C. Yu and H. Tan, RSC Adv., 2016, 6, 3840-3849.

8 K. Hung, C. Tseng, L. Dai and S. Hsu, Biomaterials, 2016, 83, 156-168.

9 Q. Chen, S. Liang and G. Thouas, Prog. Polym. Sci., 2013, 38, 584-671.

10 J. Yang, B. Chun, Y. Chung and J. Cho, Polymer, 2003, 44, 3251-3258.

11 I. Pereira and R. Oréfice, J. Mater. Sci., 2009, 45, 511-522.

12 C. Ruan, Y. Wang, M. Zhang, Y. Luo, C. Fu, M. Huang, J. Sun and C. Hu, Polym. Int., 2012, 61, 524-530.

13 X. Zhang, Y. Ma, Y. Li, P. Wang, Y. Wang and Y. Luo, Front. Mater. Sci., 2012, 6, 326-337.

14 P. Król, Prog. Mater. Sci., 2007, 52, 915-1015.

15 S. Cooper J. Guan, Advances in Polyurethane Biomaterials, Elsevier Science, 2016.

16 L. Hernández, F. Sánchez, J. Ribelles and S. Serra, J. Appl. Polym. Sci., 2011, 119, 2093-2104.

17 Y. Wang, C. Ruan, J. Sun, M. Zhang, Y. Wu and K. Peng, Polym. Degrad. Stab., 2011, 96, 1687-1694.

18 C. Ruan, N. Hu, Y. Hu, L. Jiang, Q. Cai, H. Wang, H. Pan, W. W. Lu and Y. Wang, Polymer, 2014, 55, 1020-1027.

19 C. Ruan, Y. Hu, L. Jiang, Q. Cai, H. Pan and H. Wang, J. Appl. Polym. Sci., 2014, 131, 40527.

20 F. Fenouillot, A. Rousseau, G. Colomines, R. Saint-Loup and J. Pascault, Prog. Polym. Sci., 2010, 35, 578-622.

21 M. Hellman, T. Bowmer and G. Taylor, Macromolecules, 1983, 16, 34-38. 
22 H. Marrs, D. Barton, C. Doyle, R. Jones, E. Lewis, I. Ward and J. Fisher, J. Mater. Sci.: Mater. Med., 2001, 12, 621-628.

23 T. Saito, J. Perkins, D. Jackson, N. Trammel, M. Hunt and A. Naskar, RSC Adv., 2013, 3, 21832-21840.

24 F. Chowdhury, S. Na, D. Li, Y. Poh, T. Tanaka, F. Wang and N. Wang, Nat. Mater., 2010, 9, 82-88.

25 M. Sun, A. Liu, C. Ma, H. Shao, M. Yu, Y. Liu, S. Yan and Z. Gou, RSC Adv., 2016, 6, 586-596.

26 J. van Velthoven, L. Gootjes, B. Noordover and J. Meuldijk, Eur. Polym. J., 2015, 66, 57-66.

27 T. Gnanarajan, N. Iyer, A. Nasar and G. Radhakrishnan, Eur. Polym. J., 2002, 38, 487-495.

28 J. Liu and D. Ma, J. Appl. Polym. Sci., 2002, 84, 2206-2215.
29 C. Lin, S. Kuo, C. Huang and F. Chang, Polymer, 2010, 51, 883-889.

30 Y. He, B. Zhu and Y. Inoue, Prog. Polym. Sci., 2004, 29, 10211051.

31 M. Selvakumar, H. Pawar, N. Francis, B. Das, S. Dhara and S. Chattopadhyay, ACS Appl. Mater. Interfaces, 2016, 8, 5941-5960.

32 J. C. Middleton and A. Tipton, Biomaterials, 2000, 21, 23352346.

33 S. Yang, Z. Wu, W. Yang and M. Yang, Polym. Test., 2008, 27, 957-963.

34 L. Chan, C. Tkaczyk, R. Coronado, J. Cervantes, M. Tabrizian and J. Cauich, J. Mater. Sci.: Mater. Med., 2013, 24, 17331744. 